\title{
La laparotomía de control de daños en pacientes sin trauma reduce el número de ostomías
}

\author{
Damage control laparotomy in non-trauma patients reduces the number \\ of ostomies
}

\begin{abstract}
Juan José Meléndez ${ }^{1}$ D, Claudia Patricia Orlas², Juan Pablo Herrera-Escobar², Mónica Guzmán ${ }^{3} \mathbb{D}$, Kimberly Alcázar ${ }^{4}$, Juan José Martínez ${ }^{4}$, María Fernanda Martínez ${ }^{4} \mathbb{D}$, Juan Pablo Villegas, ${ }^{4}$ José J. Serna ${ }^{5}$, Alexander Salcedo ${ }^{5}$, Leonel Leal ${ }^{1}$, Edison Angamarca1 ${ }^{1 D}$, Juliana Ordóñez ${ }^{1}$ D, Alberto F. García ${ }^{5}$ D, Michael W. Parra ${ }^{6}$, Carlos A. Ordóñez ${ }^{7}$ iD

Fellowship de Cirugía de Trauma y Emergencias, Universidad del Valle, Cali, Colombia

2 Center for Surgery and Public Health, Department of Surgery, Brigham and Women's Hospital, Harvard Medical School and Harvard T. H. Chan School of Public Health, Boston, MA, USA

Centro de Investigaciones Clínicas, Fundación Valle del Lili, Cali, Colombia

4 Facultad de Ciencias de la Salud, Universidad Icesi, Cali, Colombia

Departamento de Cirugía de Trauma y Emergencias, Fundación Valle del Lili-Universidad del Valle, Cali, Colombia

Department of Trauma and Acute Care Surgery, Broward General Level I Trauma Center, Fort Lauderdale, FL, USA

7 Jefe, Sección Cirugía de Trauma y Emergencias, Fundación Valle del Lili; director, de los fellowships en Cirugía de Trauma y Emergencias, Universidad del Valle, Cali, Colombia
\end{abstract}

\section{Resumen}

Introducción. El objetivo de este estudio fue evaluar si la laparotomía de control de daños con ligadura y reconstrucción intestinal diferida, en pacientes con peritonitis secundaria a compromiso de víscera hueca, reduce el número de ostomías.

Métodos. Se incluyeron todos los pacientes menores de I8 años de edad que ingresaron a la clínica con sospecha de peritonitis de origen no traumático y que se sometieron a laparotomía entre enero del 2003 y diciembre del 20I8. Se evaluaron las características sociodemográficas, comorbilidades, escalas de gravedad fisiológica, técnicas de reconstrucción intestinal y resultados clínicos.

Resultados. Se incluyeron 306 pacientes, distribuidos en tres grupos: I) I20 (39,2 \%) sometidos a resección y anastomosis, 2) 87 (28,4 \%) sometidos a ostomía, y 3) 99 (32,3\%) sometidos inicialmente a ligadura intestinal. Los pacientes sometidos a ligadura intestinal presentaron mayor compromiso fisiológico al ingreso a la unidad de cuidado intensivo, con puntuación APACHE II: I4 (rango intercuartílico, RIC=IO-I8) en el grupo I, I3 (RIC=II-I8) en el grupo 2, y I8 (RIC=I4-24) en el grupo 3 (p<0,oI). Sin embargo, más de la mitad se reconstruyeron en la siguiente laparotomía: anastomosis mecánica (I6/99; I6,I \%), anastomosis manual (49/99; 49,5\%), ostomía (34/99; 34,3\%).

Fecha de recibido: 31/06/2019 - Fecha de aceptación: 21/08/2019

Correspondencia: Carlos Alberto Ordóñez, Carrera 98 N 18-49, Fundación Valle del Lili, Cali, Colombia. Teléfono: (300) 631-9118 Correo electrónico: ordonezcarlosa@gmail.com

Citar como: Meléndez JJ, Orlas CP, Herrera-Escobar JP, Guzmán M, Alcázar K, Martínez JJ, et al. La laparotomía de control de daños en pacientes sin trauma reduce el número de ostomías. Rev Colomb Cir. 2020;35:455-63. https://doi.org/10.30944/20117582.727

Este es un artículo de acceso abierto bajo una Licencia Creative Commons - BY-NC-ND https://creativecommons.org/licenses/by-ncnd/4.0/deed.es 
Además, estos pacientes sometidos a ligadura intestinal tuvieron un número significativamente mayor de nuevas laparotomías, y de días de asistencia respiratoria mecánica, de estancia en la unidad de cuidado intensivo y de estancia hospitalaria. No hubo diferencias estadísticamente significativas en la mortalidad entre los subgrupos: (grupo I=I9 (I5,8 \%), grupo 2=I6 (I8,4\%), grupo 3=I9 (I9,2\%) (p=0,79).

Conclusión. En este estudio, se logró evitar la ostomía como técnica de reconstrucción definitiva en más de la mitad de los pacientes con peritonitis que se sometieron a laparotomía de control de daños con ligadura intestinal.

Palabras clave: laparotomía; control del daño; reanimación; peritonitis; estomía.

\begin{abstract}
Introduction. The objective of this study was to evaluate if the damage control laparotomy with ligation and delayed intestinal reconstruction (DR), in patients with peritonitis secondary to compromised hollow viscera, reduces the number of ostomies.

Methods. All patients under I8 years of age who entered the clinic with suspected non-traumatic peritonitis and who underwent laparotomy between January 2003 and December 2018 were included. Sociodemographic characteristics, comorbidities, severity scales physiological, intestinal reconstruction techniques and clinical results were evaluated.

Results. A total of 306 patients were included, divided into three groups: I) I2O (39.2 \%) underwent resection and anastomosis, 2) 87 (28.4\%) underwent ostomy, and 3) 99 (32.3\%) underwent initial to intestinal ligation. Patients undergoing intestinal ligation presented greater physiological compromise upon admission to the intensive care unit, with an APACHE II score: I4 (interquartile range, IQR= IO-I8) in group I, I3 (IQR = II-I8) in the group 2, and I8 (IQR = I4-24) in group $3(\mathrm{p}<\mathrm{O} .0 \mathrm{I})$. However, more than half were reconstructed in the following laparotomy: mechanical anastomosis (I6/99; I6.I \%), manual anastomosis (49/99; 49.5\%), ostomy (34/99;34.3\%). Also, they had a significantly greater number of new laparotomies, and of days of mechanical respiratory assistance, of stay in the intensive care unit and of hospital stay. There were no statistically significant differences in mortality between the subgroups: group $\mathrm{I}=\mathrm{I} 9(\mathrm{I} 5.8 \%)$, group $2=\mathrm{I} 6(\mathrm{I} 8.4 \%)$, group $3=\mathrm{I} 9(\mathrm{I} 9.2 \%)(\mathrm{p}=0.79)$.
\end{abstract}

Conclusion. In this study, it was possible to avoid the ostomy as the definitive reconstruction technique in more than half of the patients with peritonitis who underwent damage control laparotomy with intestinal ligation.

Key words: laparotomy; damage control; resuscitation; peritonitis; ostomy.

\section{Introducción}

La cirugía de control de daños se ha establecido como tratamiento para los pacientes críticamente enfermos con lesiones traumáticas del abdomen y otras cavidades ${ }^{\mathrm{I}-4}$. Las estrategias de este tipo de cirugía incluyen una laparotomía abreviada seguida del traslado inmediato a la unidad de cuidados intensivos para la reanimación fisiológica y un reparo quirúrgico definitivo diferido a un segundo tiempo en sala de operaciones 5,6 . La base fisiológica para recurrir a la cirugía de control de daños es la necesidad de interrum- pir el círculo vicioso de acidosis, hipotermia y coagulopatía, presente en pacientes de trauma, el cual implica el agotamiento de la reserva fisiológica y conlleva la muerte ${ }^{7,8}$. La introducción de dicha cirugía fue un cambio del paradigma en el manejo del trauma grave y ha representado un aumento significativo en la supervivencia de los pacientes críticamente enfermos. Actualmente, es el tratamiento estándar en los centros especializados en trauma a nivel mundial 9 .

Como los pacientes de cirugía general y de cirugía de urgencia que sufren infección intra- 
peritoneal grave o sangrado están expuestos a experimentar los efectos de la acidosis, la hipotermia y la coagulopatía, los cirujanos tratantes han usado gradualmente los principios de la cirugía de control de daños en aquellos con enfermedades de origen no traumático que presentan grave compromiso fisiológico y anatómico ${ }^{\mathrm{I}, \mathrm{II}}$. Recientemente, ha aumentado el número de publicaciones que muestran resultados prometedores en casos de isquemia mesentérica aguda, peritonitis secundaria a perforación de víscera hueca, peritonitis posquirúrgica, pancreatitis aguda, enterocolitis necrosante, hemorragia o síndrome de compartimiento abdominal ${ }^{12-16}$. En la mayoría de ellas, se reportan series heterogéneas con muestras pequeñas de pacientes con un rango variado de condiciones; algunas veces, incluyen pacientes tratados con abdomen abierto y laparotomía diferida para reparo quirúrgico definitivo, en ausencia de criterios para cirugía de control de daños ${ }^{8}$. La combinación de todos estos factores dificulta el análisis y la interpretación de los resultados.

Por lo anterior, es importante evaluar las indicaciones, las técnicas quirúrgicas y los resultados clínicos en pacientes con enfermedad intraabdominal de origen no traumático sometidos a laparotomía de control de daños, agrupándolos en varias categorías según el diagnóstico clínico e intraoperatorio (perforación, isquemia mesentérica) y condición fisiopatológica (hemorragia, sepsis), para contribuir a mejorar y hacer más eficientes los criterios que caracterizan los que podrían obtener mejores resultados con dicha cirugía.

El objetivo de este estudio fue evaluar si la laparotomía de control de daños con ligadura y reconstrucción intestinal diferida, reduce el número de ostomías en pacientes con peritonitis secundaria a compromiso de víscera hueca.

\section{Métodos}

\section{Diseño de estudio}

Este fue un estudio retrospectivo de cohorte, aprobado por el Comité de Ética de la Fundación Valle del Lili, un centro de trauma de nivel I con gran volumen de pacientes, ubicado en el suroccidente colombiano, que cuenta con un equipo multidisciplinario conformado por el Servicio de Cirugía General, el de Cirugía de Trauma y el de Cirugía de Emergencias.

\section{Población}

Se consideraron todos los pacientes adultos, mayores de 18 años de edad, que ingresaron a la institución entre enero del 2003 y diciembre del 20I8, y fueron sometidos a laparotomía de urgencia con diagnóstico de peritonitis.

Los criterios de inclusión fueron:

I. diagnóstico de peritonitis secundaria a compromiso de víscera hueca y

2. uso de alguna técnica de reparo intestinal (anastomosis manual o mecánica, sutura primaria), ostomía (ileostomía o colostomía) o ligadura intestinal con reconstrucción definitiva diferida, durante la laparotomía índice o inicial.

Los criterios de exclusión fueron:

I. peritonitis secundaria a trauma,

2. peritonitis de origen biliar,

3. peritonitis secundaria a pancreatitis,

4. laparotomía por sangrado (aneurisma de aorta abdominal roto, hemorragia de vías digestivas),

5. isquemia mesentérica,

6. peritonitis manejadas con lavado y abdomen abierto, sin técnica de reparo intestinal,

7. muerte antes de la reconstrucción intestinal, y

8. ausencia de información sobre la estrategia de reconstrucción intestinal.

\section{Estrategias de manejo quirúrgico}

Los 306 pacientes se dividieron en tres grupos, según la estrategia de manejo usada durante la laparotomía inicial: 
I. reparo definitivo, es decir, reparo primario o resección y anastomosis intestinal,

2. ostomía y reparo diferido, o

3. ligadura intestinal y reconstrucción definitiva diferida.

En el Departamento de Cirugía se encuentran estandarizadas las siguientes consideraciones para seleccionar los pacientes que se consideran de alto riesgo y que, por lo tanto, requieren reparo diferido: signos de hipoperfusión intestinal, puntaje de 15 o más en la escala APACHE II, y comorbilidades como diabetes, enfermedad cardiaca o cáncer; además, uno o más de los siguientes parámetros: respiratorio $\left(\mathrm{PO}_{2} / \mathrm{FiO}_{2}\right.$ $<200$ ), renal (creatinina sérica:3,5-4,9 mg/dl) o hepático (bilirrubina: 6-II,9 $\mathrm{mg} / \mathrm{dl}$ ), presión arterial media menor de $70 \mathrm{~mm} \mathrm{Hg}$ durante la cirugía, necesidad de vasopresor, y compromiso neurológico (Glasgow $<9$ ), trombocitopenia (plaquetas $<50.000 \mathrm{~mm}^{3}$ ) o ambos.

En el grupo I, el de reparo definitivo, después del drenaje o lavado de la cavidad peritoneal y del desbridamiento o la resección del tejido necrótico o infectado, la peritonitis se controló mediante resección del segmento intestinal comprometido y restauración de la función gastrointestinal durante la laparotomía índice con anastomosis primaria u ostomía primaria (grupo 2). Para el manejo de la pared abdominal, se utilizó el cierre definitivo o un sistema temporal de abdomen abierto según el criterio del cirujano tratante. Según consenso de los cirujanos institucionales, las indicaciones de abdomen abierto son la infección intraabdominal persistente y como prevención del síndrome de compartimiento abdominal.

En el grupo 3, el de ligadura intestinal y reconstrucción definitiva diferida, después del control inicial de la infección con lavado o drenaje de la cavidad peritoneal y con desbridamiento o resección del tejido necrótico o infectado, se practicó la resección intestinal y se ligaron los extremos proximal y distal. Una vez se lograba la estabilidad hemodinámica del paciente y se consideraba factible la reconstrucción intestinal, se llevaba a cabo la reconstrucción definitiva diferida, fuera mediante anastomosis u ostomía.

\section{Análisis estadístico}

Se describieron las características demográficas, el estado basal de ingreso, la gravedad anatómica o fisiológica y los resultados clínicos. Se utilizaron la mediana y el rango intercuartílico (RIC) para las variables continuas con distribución no normal y, la media y la desviación estándar, para las variables continuas con distribución normal. Las variables continuas se compararon utilizando pruebas no paramétricas (Kruskal-Wallis) o pruebas paramétricas (Anova), dependiendo de su distribución. Las variables categóricas se resumieron utilizando frecuencias absolutas y porcentajes, y se compararon usando la prueba de ji al cuadrado o la exacta de Fisher, cuando las frecuencias esperadas de las celdas estuvieron por debajo de 5. Los valores de p menores de 0,05 se consideraron estadísticamente significativos. Todos los análisis se llevaron a cabo en el software Stata/MP ${ }^{\mathrm{TM}}$, versión I4.O (StataCorp, College Station, TX).

\section{Resultados}

Se identificaron 708 pacientes con diagnóstico de abdomen agudo sometidos a laparotomía de urgencia. De estos, se excluyeron 8 a quienes, después de la laparotomía, se les diagnosticó isquemia mesentérica incompatible con la vida $y$, por lo tanto, no requirieron una estrategia de manejo adicional. Otros excluidos fueron: 299 pacientes en quienes, después de la laparotomía, la peritonitis solo se manejó con lavado de la cavidad peritoneal y abdomen abierto; II con ligadura durante la laparotomía índice pero que murieron antes de poder hacer la reconstrucción, y 84 cuyos datos sobre la técnica de reconstrucción intestinal estaban incompletos.

Los 306 pacientes restantes se incluyeron en el análisis. Se agruparon según el manejo recibido durante la laparotomía inicial, en: grupo I (I20; 39,2 \%), con reparo definitivo, es decir, reparo 
primario o resección y anastomosis intestinal; grupo $2(87 ; 28,4 \%)$, con ostomía y reparo diferido, y grupo $3(99 ; 32,3 \%)$, con ligadura intestinal y reconstrucción definitiva diferida (figura I).

La mayoría (I77; 57,8 \%) de los pacientes incluidos eran hombres y se encontraban en la séptima década de la vida (64 años; RIC $=48-73$ ). La comorbilidad que presentaron con mayor frecuencia estos pacientes fue el cáncer, con una proporción mayor entre los sometidos a ostomía durante la laparotomía índice: $43(35,8 \%)$ del grupo I, $46(52,9 \%)$ del grupo 2 y $24(24,2 \%)$ del grupo 3 (p<0.ooI); le siguieron la enfermedad cardiaca
(70; 22,8 \%), la diabetes ( $44 ; 14,4 \%$ ) y enfermedad renal crónica (30; 9,8\%), sin diferencias estadísticamente significativas entre los grupos para estas últimas (tabla I).

Los pacientes a quienes se les practicó ligadura intestinal tuvieron mayor puntaje en el Apache II al ingreso a la unidad de cuidado intensivo: grupo I: I4 (RIC: IO-I8); grupo 2: I3 (RIC: II-I8); grupo 3: I8 (RIC: I4-24); (p<0,ooI). Sin embargo, aquellos con reparo primario o resección y anastomosis durante la laparotomía índice, requirieron de manera significativa mayor volumen de transfusión de glóbulos rojos [grupo I: 4 (RIC: 2-7);

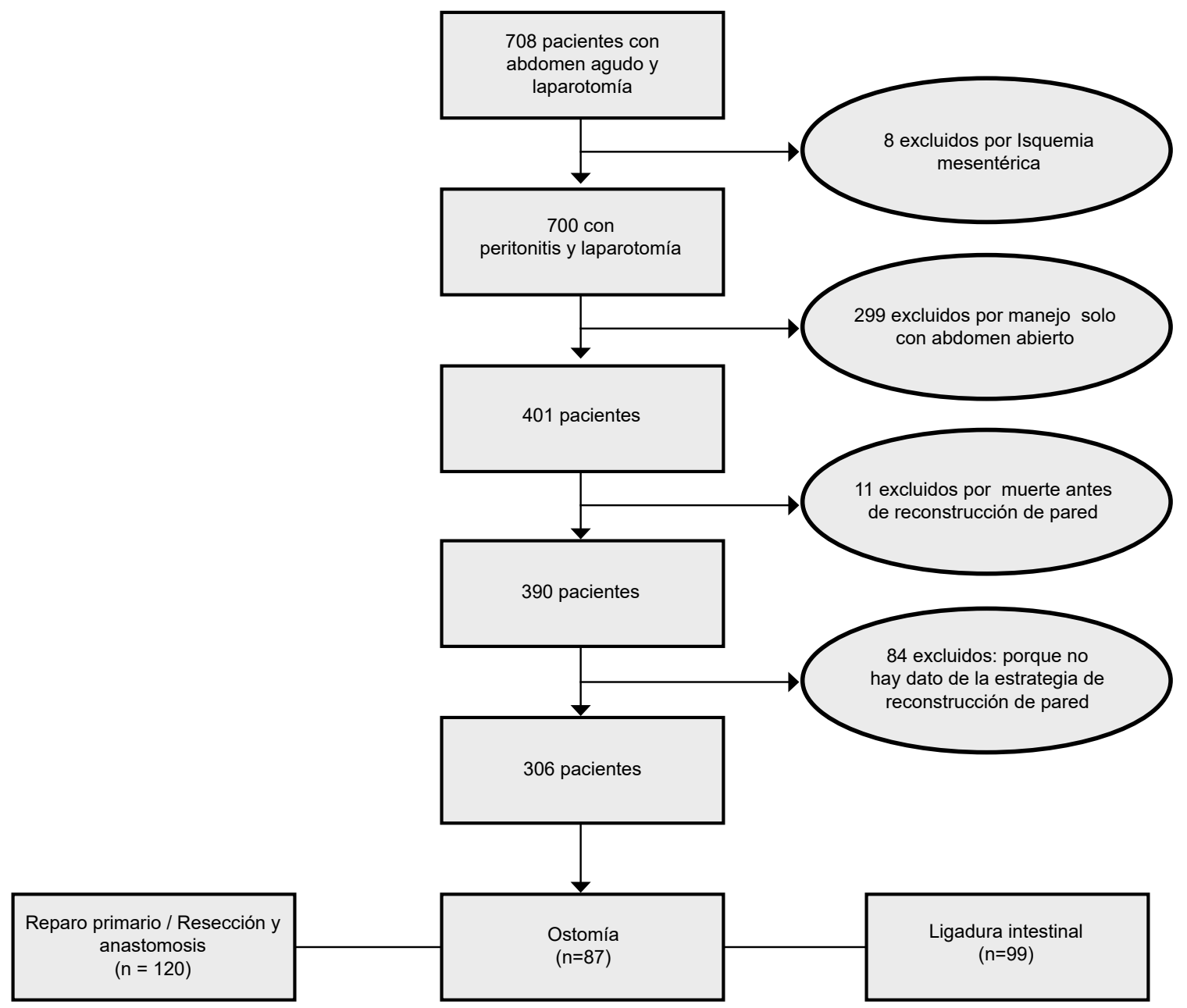

Figura 1. Distribución de los pacientes con diagnóstico de abdomen agudo sometidos a laparotomía de urgencia. 
Tabla 1. Variables sociodemográficas, escalas de severidad, componentes de reanimación y comorbilidades de los pacientes incluidos en el estudio.

\begin{tabular}{|c|c|c|c|c|c|}
\hline Variables & $\begin{array}{c}\text { Total } \\
(n=306)\end{array}$ & $\begin{array}{c}\text { Reparo primario } / \\
\text { resección y anastomosis } \\
(n=120)\end{array}$ & $\begin{array}{c}\text { Ostomía } \\
(n=87)\end{array}$ & $\begin{array}{l}\text { Ligadura intestinal } \\
\qquad(n=99)\end{array}$ & $\mathbf{p}$ \\
\hline Edad, mediana $(\mathrm{RIQ})^{*}$ & $64(48-73)$ & $62(46-73)$ & $64(51-76)$ & $64(49-74)$ & 0,32 \\
\hline Sexo masculino, n (\%) & $117(57,8)$ & $69(57,5)$ & $56(64,4)$ & $52(52,5)$ & 0,26 \\
\hline $\begin{array}{l}\text { Puntaje APACHE-II al ingreso a } \\
\mathrm{UCl} \text {, mediana (RIQ) }\end{array}$ & $15(11-20)$ & $14(10-18)$ & $13(11-18)$ & $18(14-24)$ & $<0,001$ \\
\hline $\begin{array}{l}\text { Puntaje SOFA* al ingreso a UCl, } \\
\text { mediana (RIQ) }\end{array}$ & $6(2-13)$ & $4(1-14)$ & $6(2-13)$ & $7(3-11)$ & 0,06 \\
\hline UGR* en 24 horas, mediana (RIQ) & $4(2-7)$ & $4(2-7)$ & $3(2-7)$ & $4(2-6)$ & 0,001 \\
\hline UPFC* en 24 horas, mediana (RIQ) & $4(3-8)$ & $4(3-8)$ & $7(4-12)$ & $4(3-7)$ & 0,02 \\
\hline \multicolumn{6}{|l|}{ Comorbilidades } \\
\hline Diabetes, n (\%) & $44(14,4)$ & $21(17,5)$ & $11(12,6)$ & $12(12,1)$ & 0,46 \\
\hline Enfermedad cardiaca, n (\%) & $70(22,8)$ & $23(19,2)$ & $18(20,7)$ & $29(29,3)$ & 0,17 \\
\hline Cáncer, n (\%) & $113(36,9)$ & $43(35,8)$ & $46(52,9)$ & $24(24,2)$ & $<0,001$ \\
\hline Enfermedad renal crónica, $\mathrm{n}(\%)$ & $30(9,8)$ & $17(14,2)$ & $5(5,7)$ & $8(8,1)$ & $<0,10$ \\
\hline
\end{tabular}

* RIQ: rango intercuartílico, UCl: Unidad de Cuidados Intensivos, SOFA: Sequential Organ Failure Assessment, UGR: unidades de glóbulos rojos, UPFC: unidades de plasma fresco congelado.

grupo 2:3 (RIC: 2-7); grupo 3: 4 (RIC: 2-6); $\mathrm{p}=0,00 \mathrm{O}$ ] durante las primeras 24 horas.

La peritonitis secundaria fue la condición patológica con mayor representación (26I, 85,3\%) entre los pacientes incluidos. En la mayoría de los casos, la peritonitis fue: generalizada, en 96 (80 \%) del grupo I, en $65(74,7 \%)$ del grupo 2 y en $88(88,9 \%)$ del grupo $3(\mathrm{p}=0,04)$; purulenta, en 93 $(77,5 \%)$ del grupo I, en $63(72,4 \%)$ del grupo 2 , y en $68(69,4 \%)$ del grupo $3(\mathrm{p}=0,39)$; y posquirúrgica, en 74 (6I,7\%) del grupo I, en 53 (6I,0 \%) del grupo 2 y en $45(45,4 \%)$ del grupo $3(\mathrm{p}=0,03)$ (tabla 2$)$.

La proporción de reintervenciones con laparotomía fue significativamente mayor entre los pacientes a quienes se les practicó ligadura intestinal: grupo I: I (RIC: O-2); grupo 2: I (RIC: o-3); grupo 3:3 (RIC: $\mathrm{I}-4)$; ( $\mathrm{p}<\mathrm{O}, \mathrm{OOI}$ ). Sin embargo, a menos de dos tercios de los pacientes se les hizo un cierre definitivo de la fascia al final de la hospitalización: a $62(5 \mathrm{I}, 7 \%)$ del grupo I, a $36(4 \mathrm{I}, 4 \%)$ del grupo 2 y a 6I $(6 \mathrm{I}, 6 \%)$ del grupo $3(\mathrm{p}=0,02)$. Además, los pacientes con ligadura requirieron más días de asistencia respiratoria mecánica, de estancia en la unidad de cuidados intensivos y de estancia hospitalaria, con diferencias estadísticamente significativas con respecto a los demás subgrupos (tabla 3 ).

Respecto a la técnica de la reconstrucción intestinal después de la laparotomía índice, en menos de la mitad (34/99; 34,3\%) de los pacientes se utilizó la ostomía, en 49 (49,5\%), la anastomosis manual y, en 34 (34,3\%), la anastomosis mecánica. Murieron 54 ( $17,6 \%)$ pacientes, sin diferencias estadísticamente significativas según los grupos: I9 (I5,8 \%) del grupo I, I6 (I8,4 \%) del grupo 2, y I9 $(19,2 \%)$ del grupo $3(\mathrm{p}=0,79)$.

\section{Discusión}

La hipótesis de este estudio era que la laparotomía de control de daños en pacientes con peritonitis de origen no traumático y secundaria a compromiso de víscera hueca, podría reducir el número de ostomías.

Los hallazgos sugieren que, en pacientes con múltiples comorbilidades, peritonitis purulenta o fecal, de tipo generalizada y, con grave compromiso fisiológico, es factible utilizar la ligadura 
Tabla 2. Clasificación de la peritonitis en los pacientes incluidos en el estudio.

\begin{tabular}{|c|c|c|c|c|c|}
\hline Variables & $\begin{array}{c}\text { Total } \\
(n=306)\end{array}$ & $\begin{array}{c}\text { Reparo primario / resección } \\
\text { y anastomosis } \\
(n=120)\end{array}$ & $\begin{array}{c}\text { Ostomía } \\
(n=87)\end{array}$ & $\begin{array}{c}\text { Ligadura } \\
\text { intestinal } \\
(n=99)\end{array}$ & $\mathbf{p}$ \\
\hline \multicolumn{6}{|l|}{ Según origen, $n(\%)$} \\
\hline Peritonitis primaria & $3(0,9)$ & $0(0)$ & $1(1,1)$ & $2(2,0)$ & \multirow{3}{*}{0,03} \\
\hline Peritonitis secundaria & $261(85,3)$ & $110(91,7)$ & $74(85,1)$ & $77(77,8)$ & \\
\hline Peritonitis terciaria & $42(13,7)$ & $10(8,3)$ & $12(13,8)$ & $20(20,2)$ & \\
\hline \multicolumn{6}{|l|}{ Según localización, n (\%) } \\
\hline Peritonitis localizada & $57(18,6)$ & $24(20)$ & $22(25,3)$ & $11(11,1)$ & \multirow{2}{*}{0,04} \\
\hline Peritonitis generalizada & $249(81,4)$ & $96(80)$ & $65(74,7)$ & $88(88,9)$ & \\
\hline \multicolumn{6}{|l|}{ Según material, n (\%) } \\
\hline Peritonitis purulenta & $224(73,4)$ & $93(77,5)$ & $63(72,4)$ & $68(69,4)$ & \multirow{2}{*}{$0,3 s$} \\
\hline Peritonitis fecal & $81(26,6)$ & $27(22,5)$ & $24(27,6)$ & $30(30,6)$ & \\
\hline \multicolumn{6}{|l|}{ Según momento, n (\%) } \\
\hline Peritonitis postoperatoria & $172(56,2)$ & $74(61,7)$ & $53(61)$ & $45(45,4)$ & 0,03 \\
\hline
\end{tabular}

Tabla 3. Desenlaces clínicos de los pacientes con peritonitis sometidos a laparotomía de control de daños

\begin{tabular}{|c|c|c|c|c|c|}
\hline Variables & $\begin{array}{c}\text { Total } \\
(n=306)\end{array}$ & $\begin{array}{l}\text { Reparo primario / } \\
\text { resección y anastomosis } \\
(n=120)\end{array}$ & $\begin{array}{c}\text { Ostomía } \\
(\mathrm{n}=87)\end{array}$ & $\begin{array}{c}\text { Ligadura } \\
\text { intestinal } \\
(n=99)\end{array}$ & p \\
\hline $\begin{array}{l}\text { Número de relaparotomías, mediana } \\
(\mathrm{RIQ})^{\star}\end{array}$ & $1(1-3)$ & $1(0-2)$ & $1(0-3)$ & $3(1-4)$ & $<0,001$ \\
\hline Cierre de fascia, n (\%) & $159(51,9)$ & $62(51,7)$ & $36(41,4)$ & $61(61,6)$ & \multirow{2}{*}{0,02} \\
\hline Cierre de piel, n (\%) & $147(48)$ & $58(48,3)$ & $51(58,6)$ & $38(38,4)$ & \\
\hline $\begin{array}{l}\text { Días de ventilación mecánica, } \\
\text { mediana (RIQ) }\end{array}$ & $5(2-12)$ & $3(1-9)$ & $3(1-8)$ & $8(4-15)$ & $<0,001$ \\
\hline Estancia en $\mathrm{UCl}^{*}$, mediana (RIQ) & $12(5-22)$ & $9(3-20)$ & $10(4-16)$ & $15(10-25)$ & $<0,001$ \\
\hline Estancia hospitalaria, mediana (RIQ) & $25(14-28)$ & $23(11-36)$ & $22(14-32)$ & $31(21-46)$ & 0,001 \\
\hline Mortalidad intrahospitalaria, n (\%) & $54(17,6)$ & $19(15,8)$ & $16(18,4)$ & $19(19,2)$ & 0,79 \\
\hline
\end{tabular}

${ }^{*}$ RIQ: rango intercuartílico, UCI: Unidad de Cuidados Intensivos.

intestinal con reconstrucción diferida. Aunque este grupo requirió mayor número de reintervenciones con laparotomía, de días de asistencia respiratoria mecánica, de estancia en la unidad de cuidados intensivos y de días de estancia hospitalaria, dicha técnica evitó la ostomía en más de la mitad de los pacientes, los cuales terminaron con reconstrucción definitiva del tubo gastrointestinal antes del alta hospitalaria: 49/99 (49,5\%) con anastomosis manual diferida y $\mathrm{I} 6 / 99$ (I6,I \%) con anastomosis mecánica diferida.

En estudios previos se ha establecido que la laparotomía de control de daños es una estrategia eficaz para el manejo de pacientes con infección intraabdominal. Sin embargo, la falta de un grupo de control no ha permitido concluir si hay superioridad de este abordaje y, aunque se han propuesto varias técnicas de manejo quirúrgico, todavía falta 'evidencia' que soporte el efecto de esta en los resultados clínicos cuando se realizan procedimientos de reparo definitivo durante la laparotomía índice, en comparación con la reconstrucción definitiva después de la laparotomía abreviada ${ }^{17,18}$.

Los resultados de este estudio sustentan la evidencia existente, la cual sugiere que los pacientes con grandes probabilidades de muerte según escalas como la Apache II y la SOFA, con 
una cantidad heterogénea de comorbilidades como cáncer, enfermedad cardiaca, diabetes o enfermedad renal, podrían beneficiarse de un principio usado en la cirugía de control de daños, que es expectante. Es decir, después de un diagnóstico oportuno de la alteración intraabdominal, una laparotomía abreviada para controlar la fuente de infección, el traslado inmediato a la unidad de cuidados intensivos para la reanimación y el tratamiento complementario con antibióticos, se hace una segunda evaluación de los pacientes que ya se encuentran mejor reanimados, hidratados, con mayor estabilidad según los parámetros hemodinámicos y con control parcial de la fuente de infección.

Khan, et al, ${ }^{19}$, publicaron una serie de 42 pacientes con condiciones variadas como peritonitis, absceso intraabdominal, isquemia intestinal y sangrado, en quienes la utilización de los principios de la laparotomía de control de daños permitió el cierre temprano de la pared abdominal, lo cual se asoció con menos complicaciones adicionales.

La elección del diseño de este tipo de estudio se basó en el registro de una gran proporción de pacientes sometidos a ligadura intestinal durante la laparotomía índice. A pesar de que los estudios de tipo retrospectivo tienen limitaciones ya conocidas, cabe resaltar que, en esta cohorte de pacientes, se pudieron determinar y describir las técnicas de reconstrucción intestinal y los resultados clínicamente relevantes.

Los hallazgos de este estudio tienen solamente validez interna por las limitaciones inherentes a su diseño. Sin embargo, se ha demostrado que el uso de estas técnicas en enfermedades de naturaleza similar o en otras condiciones, como hemorragia de origen no traumático, peritonitis de origen biliar, pancreatitis necrosante sobreinfectada o tumores con perforación de víscera hueca, entre otras, es posible y tiene efectos benéficos sobre resultados clínicos como la mortalidad ${ }^{20}$.

Con base en estos resultados y considerando los potenciales beneficios de estas estrategias quirúrgicas en alteraciones de origen no traumático, se puede afirmar que es necesario continuar ampliando la evidencia disponible e implementar diseños de tipo prospectivo y estudios aleatorizados ${ }^{21}$, para determinar cuáles indicaciones podrían ser más sensibles y cuáles más específicas, en los pacientes que podrían manejarse con reconstrucción diferida; además, para establecer cuál es el efecto real de diferir la reconstrucción intestinal en la supervivencia de los pacientes.

Por último, es posible el reparo primario para el control definitivo de la infección en casos de peritonitis grave, siempre y cuando, la condición clínica y la perfusión de la víscera comprometida sean adecuadas. En otros casos, cuando la condición del paciente reviste mayor gravedad, el practicar una laparotomía abreviada y diferir el reparo podría reducir la necesidad de una ostomía como tratamiento definitivo.

En conclusión, en el presente estudio, se logró evitar la ostomía como técnica de reconstrucción definitiva en más de la mitad de los pacientes con peritonitis que se sometieron a laparotomía de control de daños con ligadura intestinal. La implementación de esta técnica en el campo de la cirugía de cuidado agudo es factible. No obstante, se requieren estudios prospectivos que establezcan las variables para predecir tempranamente cuáles pacientes se beneficiarían más con este tipo de intervención.

\section{Cumplimiento de normas éticas}

Consentimiento informado. Este es un estudio retrospectivo de cohorte, con revisión retrospectiva de historias clínicas y, como tal, no hay necesidad de un consentimiento informado. Fue aprobado por el Comité de Ética de la Fundación Valle del Lili.

Conflicto de interés. Los autores manifiestan que no tienen conflicto de intereses.

Fuentes de financiación. Los recursos propios de los autores con apoyo del Centro de Investigaciones Clínicas, de Fundación Valle del Lili, Cali, Colombia. 


\section{Referencias}

I. Stone HH, Strom PR, Mullins RJ. Management of the major coagulopathy with onset during laparotomy. Ann Surg. 1983;197:532-5. https://doi.org/IO.I097/00000658-198305000-00005

2. Rotondo MF, Schwab CW, McGonigal MD, Phillips GR, Fruchterman TM, Kauder DR, et al. Damage control: An approach for improved survival in exsanguinating penetrating abdominal injury. J Trauma. 1993;35:375-83. https://doi.org/I0.I097/00005373-199309000-00008

3. Lucas CE, Ledgerwood AM. Prospective evaluation of hemostatic techniques for liver injuries. J Trauma. I976;I6:442-5I. https://doi.org/IO.I097/00005373-I97606000-00003

4. Ordóñez CA, Badiel M, Pino LF, Salamea JC, Loaiza JH, Parra MW, et al. Damage control resuscitation: Early decision strategies in abdominal gunshot wounds using an easy "ABCD" mnemonic. J Trauma Acute Care Surg. 2012;73:1074-8.

https://doi.org/Io.I097/TA.oboi3e3I826fc78o

5. Moore E. Thomas G. Orr Memorial Lecture. Staged laparotomy for the hypothermia, acidosis, and coagulopathy syndrome. Am J Surg. 1996;172:405-I0. https://doi.org/IO.IOI6/Sooo2-96IO(96)0o2I6-4

6. Rotondo MF, Zonies DH. The damage control sequence and underlying logic. Surg Clin North Am. I997;77:76I-77.

https://doi.org/IO.IOI6/Soo39-6I09(05)70582-X

7. Gruen RL, Brohi K, Schreiber M, Balogh ZJ, Pitt V, Narayan $\mathrm{M}$, et al. Haemorrhage control in severely injured patients. Lancet. 2012;380:1099-108. https://doi.org/IO.IOI6/SoI40-6736(I2)6I224-O

8. Girard E, Abba J, Boussat B, Trilling B, Mancini A. Damage control surgery for non-traumatic abdominal emergencies. World J Surg. 2018;42:965-73. https://doi.org/I0.IO07/s00268-0I7-4262-6

9. Moore FA, McKinley BA, Moore EE. The next generation in shock resuscitation. Lancet. 2004;363:1988-96. https://doi.org/Io.IoI6/SoI40-6736(04)I64I5-5

Io. Weber DG, Bendinelli C, Balogh ZJ. Damage control surgery for abdominal emergencies. Br J Surg. 20I4;IOI:eI09-I8. https://doi.org/Io.IOO2/bjs.9360

II. Borráez O. Abdomen abierto: la herida más desafiante. Rev Colomb Cir. 2008;23:204-9.

I2. Person B, Dorfman T, Bahouth H, Osman A, Assalia A, Kluger Y. Abbreviated emergency laparotomy in the non-trauma setting. World J Emerg Surg. 2009;4:4I. https://doi.org/IO.II86/I749-7922-4-4I

I3. Ball CG, Correa-Gallego C, Howard TJ, Zyromski NJ, Lillemoe KD. Damage control principles for pancreatic surgery. J Gastrointest Surg. 2010;14:1632-3. https://doi.org/IO.I007/sII605-OIO-I286-8

I4. Filicori F, Di Saverio S, Casali M, Biscardi A, Baldoni F, Tugnoli G. Packing for damage control of non-traumatic intra-abdominal massive hemorrhages. World J Surg. 2010;34:2064-8. https://doi.org/IO.IO07/s00268-0IO-0667-I

I5. Stawicki SP, Brooks A, Bilski T, Scaff D, Gupta R, Schwab CW, et al. The concept of damage control: Extending the paradigm to emergency general surgery. Injury. 2008;39:93-IOI.

https://doi.org/I0.I0I6/j.injury.2007.06.0II

I6. Finlay IG, Edwards TJ, Lambert AW. Damage control laparotomy. Br J Surg. 2004;91:83-5. https://doi.org/IO.IOO2/bjs.4434

I7. Kafka-Ritsch R, Birkfellner F, Perathoner A, Raab H, Nehoda H, Pratschke J, et al. Damage control surgery with abdominal vacuum and delayed bowel reconstruction in patients with perforated diverticulitis Hinchey III/IV. J Gastrointest Surg. 2012;16:1915-22. https://doi.org/IO.IOO7/sII605-OI2-I977-4

I8. Cirocchi R, Arezzo A, Vettoretto N, Cavaliere D, Farinella $\mathrm{E}$, Renzi $\mathrm{C}$, et al. Role of damage control surgery in the treatment of Hinchey III and IV sigmoid diverticulitis: A tailored strategy. Medicine (Baltimore). 20I4;93:eI84. https://doi.org/I0.I097/MD.000ooooooooooI84

19. Khan A, Hsee L, Mathur S, Civil I. Damage-control laparotomy in nontrauma patients. J Trauma Acute Care Surg. 2013;75:365-8. https://doi.org/I0.I097/TA.oboi3e3I829cb65e

20. Bruns BR, Ahmad SA, Meara LO, Tesoriero R, Lauerman M, Klyushnenkova E, et al. Nontrauma open abdomens : A prospective observational study. J Trauma Acute Care Surg. 20I6;80:63I-6. https://doi.org/I0.I097/TA.0000000000000958

2I. Kirkpatrick AW, Coccolini F, Ansaloni L, Roberts DJ, Tolonen M, McKee JL, et al. Closed or open after source control laparotomy for severe complicated intra-abdominal sepsis (the COOL trial): Study protocol for a randomized controlled trial. World J Emerg Surg. 2018;13:26. 